\title{
Space-time development of in-medium hadronization : scenario for leading hadrons
}

\author{
B. Guiot ${ }^{1, a}$ and B. Kopeliovich ${ }^{1}$ \\ ${ }^{1}$ University Técnica Frederico Santa Maria (UTFSM), Valparaiso, Chile
}

\begin{abstract}
Lepton deep inelastic scattering off nuclei at medium energies gives the opportunity to study the space-time development of hadronization. Indeed, for these kinematics, the production length is comparable to the nuclear size. Based on the Berger model [1] and dipole phenomenology, we built a model for vacuum and in-medium hadronization. The model, which includes vacuum energy loss, induced energy loss and nuclear absorption in a parameter-free way successfully describes Hermes data [2, 3]. In a future publication, Fermi motion will be taken into account and the model will be applied to CLAS Eg2 data, at Jefferson laboratory.
\end{abstract}

\section{Introduction}

The study of semi-inclusive production of hadrons in deep-inelastic scattering (DIS) off nuclei is interesting for at least two reasons. It gives a better understanding of cold nuclear matter and provides information on the spacetime development of hadronization. The main observables are the hadron $p_{t}$-broadening (see section 7) and the multiplicity ratio, defined as :

$$
R_{A}\left(z, Q^{2}, E\right) \simeq \frac{1}{A} \frac{D^{A}\left(z, Q^{2}, E\right)}{D\left(z, Q^{2}, E\right)}
$$

which shows nuclear suppression (enhancement) of hadrons. Here, $D^{A}$ and $D$ are the in-medium and vacuum fragmentation functions, respectively. $z, Q^{2}$ and $E$ are the fraction of photon energy carried by the final hadron, the photon virtuality and the photon energy. Models based on induced energy loss $[4,5]$ will for instance provide information on the transport coefficient for nuclear matter $(\hat{q})$ and on the production time of the pre-hadron. Other models $[7,8]$ try to explain the nuclear suppression in terms of absorption of the (pre)hadron. ${ }^{1}$ They provide information on production and formation times as well as on the spacetime dependence of the pre-hadron cross section. Both can describe reasonably well data on DIS off nuclei which have been published by the European Muon collaboration (EMC) [6] and by the HERMES collaboration at DESY.

As underlined in [4], these models use quantities which are poorly constrained : $\hat{q}$ and the pre-hadron cross section. Consequently, it is hard to make some conclusions on the respective

\footnotetext{
a e-mail: benjamin.guiot@usm.cl

${ }^{1}$ Here and in the following, (pre)hadron stands for either the hadron or the pre-hadron.
} 
contributions of induced energy loss and nuclear absorption of the (pre)hadron to the multiplicity ratio. One of our aims is to disentangle these effects.

In this article, we present a simple model based on perturbative QCD (pQCD) for the propagation and hadronization of the leading quark in cold matter. It includes vacuum energy loss, induced energy loss and nuclear absorption. Our results are then compared to Hermes data [2, 3] (and to CLAS Eg2 data in a future publication).

\section{2 pQCD based hadronization}

Hadronization of the leading hadron is based on the Berger model [1], modified by higher order considerations [9]. In the Born approximation, the leading quark emits a gluon which splits into a $q \bar{q}$ pair. Then the $\bar{q}$ and the leading quark form the leading hadrons, as depicted in figure 1.

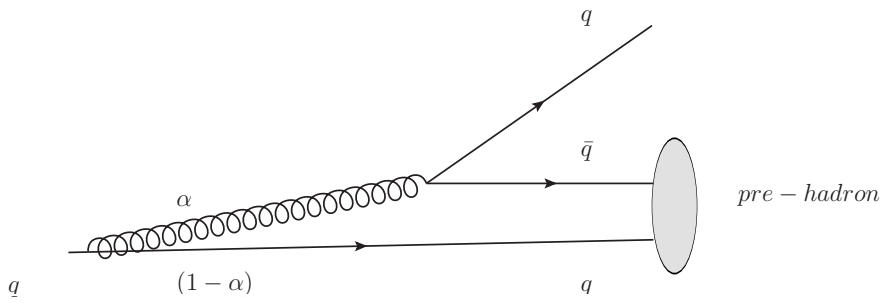

Figure 1. Berger mechanism of leading pion production in Born approximation. there, $\alpha$ is the fraction of quark energy taken by the gluon.

In this description of hadronization and in the limit $(1-z) \ll 1, k_{t} \ll Q^{2}$, one obtains the following fragmentation function for pion :

$$
\frac{\partial D^{\text {Born }}}{\partial k_{t}}\left(z, k_{t}\right) \propto \frac{(1-z)^{2}}{k_{t}^{4}}
$$

with $k_{t}$ the gluon transverse momentum relative to the leading quark direction. As explained in [9], taking into account energy loss (see figure 2), we obtain the following expression for the pion fragmentation function at large $\mathrm{z}$ :

$$
\frac{\partial D}{\partial k_{t}}\left(z, k_{t}\right) \propto \frac{(1-\tilde{z})^{2}}{k_{t}^{4}}
$$

The energy loss results in a shift of the fragmentation variable $z$ :

$$
\tilde{z}=\frac{z}{1-\Delta E / E}
$$

with $\Delta E$ being the total energy loss. In the assumption that the $q \bar{q}$ pair shares equally the gluon energy, we can write :

$$
z=\frac{E_{\pi}}{E}=\frac{(\tilde{\alpha} / 2+1-\tilde{\alpha})(E-\Delta E)}{E}=(-\tilde{\alpha} / 2+1)(1-\Delta E / E)
$$

where $\tilde{\alpha}$ is the fraction of $(E-\Delta E)$ taken by the gluon. Reversing this equation we have :

$$
\tilde{\alpha}=2\left(1-\frac{z}{1-\Delta E / E}\right)=2(1-\tilde{z})
$$




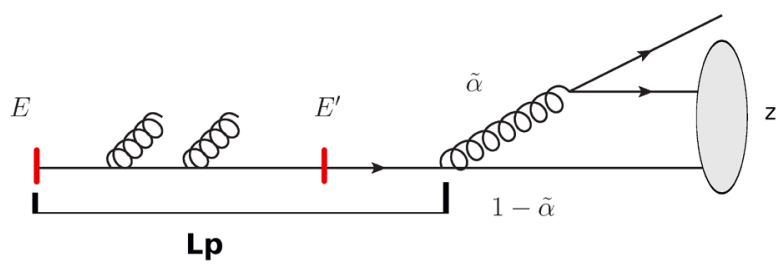

Figure 2. Berger mechanism with energy loss $\Delta E=E-E^{\prime}$. $\tilde{\alpha}$ is the quark energy fraction taken by gluon after energy loss $\Delta E$.

One can see that for $\tilde{\alpha} \in[0,1]$, we have $z \in[0.5,1]$. It comes from our assumption of equal sharing for the $q \bar{q}$ pair, but this is not an issue since we want to focus on high $z$ values. Another assumption of our model is to identify the production length of the pre-hadron with the radiation time of the gluon :

$$
L p=\frac{2 \tilde{\alpha} E^{\prime}}{k_{t}^{2}}=\frac{4 E^{\prime}(1-\tilde{z})}{k_{t}^{2}}
$$

Energy loss gives a shorter production length $(\tilde{z}>z)$ and the effect is larger for smaller value of $z$. For $z=1$, there is no energy loss allowed and we find back the result given by the Born approximation. In terms of production length, the fragmentation function, eq. (3), is given by :

$$
\frac{\partial D}{\partial L p}\left(z, E, Q^{2}, L p\right) \propto(1-\tilde{z})
$$

\section{Vacuum energy loss}

There is a perturbative and non-perturbative contribution. The perturbative one is given by :

$$
\Delta E_{p e r t}\left(L, z, Q^{2}\right)=E \int_{\lambda^{2}}^{Q^{2}} d q_{t}^{2} \int_{0}^{1} d \beta \beta \frac{d n_{g}}{d q_{t}^{2} d \beta} \Theta\left(L-l_{c}^{g}\right) \Theta(1-z-\beta)
$$

$\lambda=0.7 \mathrm{GeV}$ is a cut-off (see [9]), $q_{t}$ the gluon transverse momentum and $\beta$ is the energy fraction taken by the radiated gluon. The last step function maintains energy conservation; none of the emitted gluons can have energy larger than $(1-z) E$. The first step function takes into account gluons radiation time :

$$
l_{c}^{g}=\frac{2 \beta E}{q_{t}^{2}}
$$

and $\frac{d n_{g}}{d q_{t}^{2} d \beta}$ is the usual gluon number distribution (see [9] for more details). In terms of gluon radiation time, the perturbative energy loss is :

$$
\Delta E_{\text {pert }}\left(L, z, Q^{2}\right)=E \int_{\lambda / E}^{1-z} d \beta \int_{l_{\min }}^{l_{\max }} \beta \frac{d n_{g}}{d l d \beta} d l
$$

with boundaries for integration given by :

$$
l_{\min }=\frac{2 E \beta}{Q^{2}}, l_{\max }=\min \left[\frac{2 E \beta}{\lambda^{2}}, L\right]
$$


Due to energy conservation, energy loss goes to zero when $z \rightarrow 1$. For $L>L_{\text {max }}$, the maximal length for perturbative energy loss given by :

$$
L_{\max }=\frac{2 E(1-z)}{\lambda^{2}}
$$

the perturbative energy loss is a constant of $L$.

Non-perturbative energy loss due to color flux tube formation is based on the second model in [9]. That is :

$$
\Delta E_{n p}(L, z)=\kappa \frac{\lambda^{2} L^{2}}{E} \Theta\left(L_{\max }-L\right)+\left[\kappa \frac{\lambda^{2} L_{\max }^{2}}{E}+\kappa\left(L-L_{\max }\right)\right] \Theta\left(L-L_{\max }\right)
$$

where $\kappa=1 \mathrm{GeV} / \mathrm{fm}$ is the string tension.

\section{Fragmentation function}

In order to compare with data, one has to integrate the differential fragmentation function (8) over the production length $L p$ :

$$
D\left(z, Q^{2}, E\right) \propto \int_{L p_{\min }}^{L p_{\max }} d L p \frac{\partial D}{\partial L p}\left(z, Q^{2}, E, L p\right)
$$

with $L p_{\min }$ and $L p_{\max }$ given by the equations :

$$
L p_{\min }=\frac{2 E^{\prime}\left(1-\tilde{z}\left(L p_{\min }\right)\right)}{Q^{2}} ; L p_{\max }=\frac{2 E^{\prime}\left(1-\tilde{z}\left(L p_{\max }\right)\right)}{\lambda^{2}}
$$

Since $\tilde{z}<z$, we see that the production length is shorted by energy loss. These equations are solved numerically.

\section{In medium hadronization}

At first approximation, we are taking into account only nuclear absorption. Then the nuclear fragmentation function is simply the convolution of the vacuum fragmentation function, equation (8), with a suppression factor :

$$
D^{A}\left(z, Q^{2}, E\right) \propto \int d^{2} b \int d z_{l} \rho\left(b, z_{l}\right) \int_{L p_{\min }}^{L p_{\max }} d L p \frac{\partial D}{\partial L p}\left(z, Q^{2}, E, L p\right) \operatorname{Tr}\left(z, Q^{2}, E, b, z_{l}+L p, \infty\right)
$$

Here $b$ is the two dimensional impact parameter; $\rho$ the nuclear density, taken from $[11]$; $z_{l}$ the longitudinal coordinate of the DIS process and $\operatorname{Tr}()$ the suppression factor due to dipole absorption by the nuclear medium.

At low energies, the dipole size can fluctuate and the eikonal approximation can't be used. The $q \bar{q}$ propagation throughout the medium is achieved with the light cone Green function $G\left(z_{2}, \overrightarrow{r_{2}}, z_{1}, \overrightarrow{r_{1}}\right)$, where $z_{1}, z_{2}$ correspond to initial and final times, respectively, and $\overrightarrow{r_{1}}, \overrightarrow{r_{2}}$ represent the initial and final dipole sizes. This Green function obeys the two dimensional light cone Schrödinger equation, described in [12] together with its solution :

$$
i \frac{d}{d z_{2}} G\left(z_{2}, \overrightarrow{r_{2}}, z_{1}, \overrightarrow{r_{1}}\right)=\left[\frac{\epsilon^{2}-\Delta_{r_{2}}}{2 p \beta(1-\beta)}+V_{q \bar{q}}\left(z 2, \overrightarrow{r_{2}}, \beta\right)\right] G\left(z_{2}, \overrightarrow{r_{2}}, z_{1}, \overrightarrow{r_{1}}\right)
$$


Using the Green function, The expression for the transparency factor is :

$$
\operatorname{Tr}\left(z, Q^{2}, E, b, z_{1}, z_{2}\right)=\left|\frac{\int d^{2} r_{1} d^{2} r_{2} \psi_{h}^{*}\left(r_{2}\right) G\left(z_{2}, r_{2}, z_{1}, r_{1}\right) \psi_{q \bar{q}}\left(r_{1}\right)}{\int d^{2} r \psi_{h}^{*}(r) \psi_{q \bar{q}}(r)}\right|^{2}
$$

For the hadronic wave function, we use a parametrization in the form of the asymptotic light-cone meson wave function [13]

$$
\begin{gathered}
\psi_{h}(r)=f(\beta) \exp \left(-a^{2}(\beta) r^{2} / 2\right) \\
a^{2}(\beta)=\frac{4\left(\beta(1-\beta)+a_{0}\right)}{\left\langle r_{\pi}^{2}\right\rangle}
\end{gathered}
$$

In [8] it is found that for the value $a_{0}=1 / 12$, the previous wave function reproduces correctly the pion mean radius squared. In our model, the light cone fraction $\beta$ of the quark inside the pion wave function is related to $z$ by

$$
\beta=\frac{2 \tilde{z}-1}{\tilde{z}}
$$

This wave function is solution of the Schrödinger equation (18) if the real part of the potential is given by :

$$
\mathfrak{R} V_{q \bar{q}}\left(z_{2}, \vec{r}, \beta\right)=\frac{a^{4}(\beta) r^{2}}{2 p \beta(1-\beta)}
$$

The imaginary part of the potential responsible for absorption is given by :

$$
\mathfrak{J} V_{q \bar{q}}\left(z_{2}, \vec{r}, \beta\right)=-\frac{\sigma_{q \bar{q}}(r)}{2} \rho_{A}\left(z_{2}\right)
$$

where $\sigma_{q \bar{q}}(r)$ is the dipole cross section with nucleon. For simplicity, we use the small $r$ expression :

$$
\sigma_{q \bar{q}}(r)=C(s) r^{2}
$$

with $\sqrt{s}$ the pre-hadron nucleon center of mass energy :

$$
s=2 z M_{p} E+M_{p}^{2}+m_{\pi}^{2}
$$

The energy dependent factor $C(s)$ is constrained since the averaged dipole cross section has to reproduce the total meson $\left(\pi^{+}\right.$in our case) cross section with nucleon

$$
\left\langle\sigma_{q \bar{q}}\right\rangle=\int d^{2} r C(s) r^{2}\left|\psi_{q \bar{q}}(r)\right|^{2}=C(s)\left\langle r_{\pi}^{2}\right\rangle=\sigma_{t o t}^{\pi p}(s)
$$

Here we use $\sigma_{\text {tot }}^{\pi p}(s)=23.6\left(s / s_{0}\right)^{0.079}+1.432\left(s / s_{0}\right)^{-0.45} \mathrm{mb}$ [14] with $s_{0}=1000 \mathrm{GeV}^{2}$ for $\sqrt{s}>2.5 \mathrm{GeV}^{2}$ and a table from Igor Strakovsky otherwise [15]. Additionally, we have the relation $\left\langle r_{\pi}^{2}\right\rangle=\frac{8}{3}\left\langle r_{\pi}^{2}\right\rangle_{e m},\left\langle r_{\pi}^{2}\right\rangle_{e m}=0.44 \mathrm{fm}^{2}$ being the pion mean charge radius squared [16].

The last ingredient for the color transparency factor, equation (19), is the dipole wave function. Since we want a continuous transition between the dipole and pion wave functions, we will use :

$$
\psi_{\bar{q} q}\left(z, Q^{2}, E, L p, r\right)=f(\beta) \exp \left(-b^{2}(\beta) r^{2} / 2\right)
$$

with

$$
b^{2}(\beta)=\frac{4\left(\beta(1-\beta)+a_{0}\right)}{\left\langle r_{q \bar{q}}^{2}\right\rangle}
$$

Then for $\left\langle r_{q \bar{q}}^{2}\right\rangle=\left\langle r_{\pi}^{2}\right\rangle$, dipole and pion wave functions are equal. 


\section{Multiplicity ratio}

We now have all the ingredients in order to compute the suppression factor $\operatorname{Tr}()$ and the multiplicity ratio defined in equation (1). Even though the dipole transverse size evolves with time, it is interesting to start with the approximation $\left\langle r_{q \bar{q}}^{2}\right\rangle=\left\langle r_{\pi}^{2}\right\rangle$ since it gives the maximal contribution of nuclear absorption. Our result for the HERMES experiment [2] is presented in figure 3.

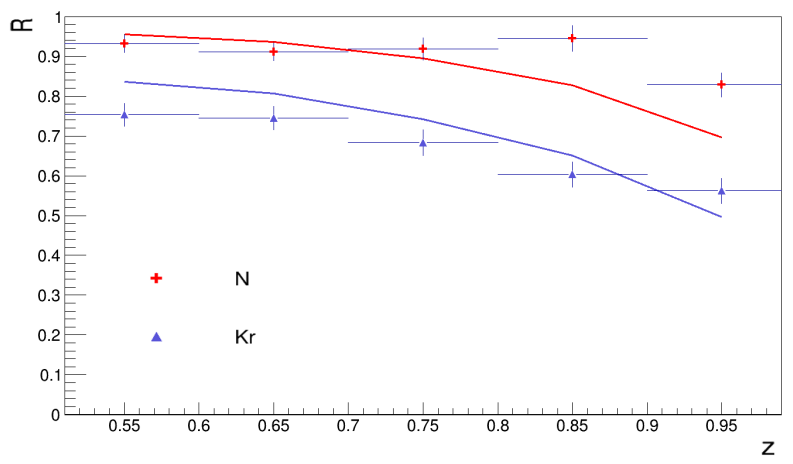

Figure 3. Multiplicity ratio for nitrogen and krypton, compared to HERMES data [2].

At $z=1$, due to energy conservation, there is no energy loss. Therefore, the suppression comes only from the nuclear absorption. Unsurprisingly, our prediction is below the data, which is the consequence of our approximation on the dipole size. This approximation was also expected to work better for big nuclei. At $z=0.5$, the fact that we are still above the data could be an indication that the contribution of induced energy loss is missing.

\section{Broadening and induced energy loss}

As it propagates through the nucleus, the leading quark undergoes multiple scattering which generates induced energy loss and $p_{t}$-broadening. Following $[17,18]$, the two main formulae are :

$$
\begin{gathered}
\delta\left\langle p_{t}^{2}\right\rangle\left(s, z_{q}, b, L\right)=2 C(s) \rho\left(z_{q}, b\right) L \\
-\frac{d E_{\text {ind }}}{d z_{q}}=\frac{3}{4} \alpha_{s} \delta\left\langle p_{t}^{2}\right\rangle
\end{gathered}
$$

where $z_{q}$ is the longitudinal coordinate of the quark. Since $\rho$ depends on $z_{q}$, the correct expression for $p_{t}$-broadening on a finite length is given by :

$$
\Delta\left\langle p_{t}^{2}\right\rangle\left(s, z_{l}, b, L_{p}\right)=2 C(s) \int_{z_{l}}^{z_{l}+L_{p}} \rho(l, b) d l
$$

where $z_{l}$ is the longitudinal coordinate of the DIS point. Total induced energy loss is given by :

$$
\Delta E_{\text {ind }}\left(s, z_{l}, b, L_{p}\right)=\frac{3}{4} \alpha_{s} \int_{0}^{L_{p}} \Delta\left\langle p_{t}^{2}\right\rangle\left(s, z_{l}, b, l\right) d l
$$


The DIS point is distributed over the whole nucleus, so we have to average equation (32) with $\frac{1}{A} \int d^{2} b d z_{1} \rho\left(z_{1}, b\right)$. We also have a distribution for the length $L_{p}$, therefore, we average equation (32) with $\frac{1}{N_{L}} \int d L_{p} \frac{\partial D}{\partial L_{p}}$. Then, for the quark, the experimental $p_{t}$-broadening is given by :

$$
\Delta\left\langle p_{t}^{2}\right\rangle_{\text {exp }}\left(z, Q^{2}, E\right)=\frac{2 C}{A} \int d^{2} b \int d z_{1} \rho\left(z_{1}, b\right) \int_{L_{\min }}^{L_{\max }} d L_{p} \frac{1}{N_{L}} \frac{\partial D}{\partial L_{p}} T_{A}\left(b, z_{1}, z_{1}+L_{p}\right)
$$

with

$$
T_{A}\left(b, z_{1}, z_{1}+L_{p}\right)=\int_{z_{1}}^{z_{1}+L_{p}} d l \rho(l, b)
$$

and the normalization

$$
N_{L}\left(z, Q^{2}, E\right)=\int_{L_{\min }}^{L_{\max }} d L_{p} \frac{\partial D}{\partial L_{p}}\left(z, Q^{2}, E, L_{p}\right)
$$

Finally, the pion $p_{t}$-broadening we are interested in is given by

$$
\Delta\left\langle k_{t}^{2}\right\rangle_{\pi}\left(z, Q^{2}, E\right)=\tilde{z}^{2} \Delta\left\langle p_{t}^{2}\right\rangle_{\exp }\left(z, Q^{2}, E\right)
$$

To take into account the induced energy loss, we need to replace $\tilde{z} \rightarrow \tilde{z}=\frac{z}{1-\left(\Delta E_{\text {vac }}+\Delta E_{\text {ind }}\right) / E}$, when computing in-medium fragmentation function $D^{A}$. Results for $p_{t}$-broadening and multiplicity ratio modified by induced energy loss are shown in Figure 4.

For $p_{t}$-broadening, our result does a reasonably good description of data. It should be noticed that HERMES do a correction (based on Monte-Carlo) for decays of the $\rho^{0}$ meson into pions. For the higher bin in $z$, more than $50 \%$ of pions are supposed to come from $\rho^{0}$ decays. This is likely an overestimation of this number which results in a negative $p_{t}$-broadening for high $z$. Notice also that one argument for the negative $p_{t}$-broadening is that the $p_{t}$ distribution of quarks inside a nucleus is larger for smaller nucleus. Then at $z=1$ (no broadening from quark multiple interaction) we could have $p_{t, A}-p_{t, \text { Deterium }}<0$. But in this case we should have $\Delta p_{t, X e}<\Delta p_{t, K r}<\Delta p_{t, N e}<0$ which is not the case.

Our result for the multiplicity ratio has been improved by induced energy loss, particularly for smaller value of $z$, as it was expected. In a future publication, we will show that the implementation of induced energy loss based on the model presented in [4] gives a similar result (using $\hat{q}=0.25$ ). At $z=0.5$, the correction of induced energy loss is of the order of $15 \%$. One of the reason why in our case, the contribution of induced energy loss appears smaller than in models based solely on this effect is probably because they use $\hat{q} \sim 0.70$ while the value for cold matter is estimated to be $\hat{q} \sim 0.25$ [18]. Another reason is that the DIS point is taken to be at the front edge of the nucleus, giving a bigger path for the quark inside nuclear matter.

\section{Conclusion}

Based on Berger's model, we can build vacuum and in-medium fragmentation functions. The suppression factor, $\operatorname{Tr}()$, which takes into account nuclear absorption of the dipole, is computed based on quantum mechanics, using an assumption for the dipole and pion waves functions. The induced energy loss has been implemented, using its relation with the $p_{t}$-broadening. With our no free parameter model, we are able to describe HERMES data for $p_{t}$-broadening and multiplicity ratio. The contribution of induced energy loss for the multiplicity ratio is found to be $15 \%$ at $z=0.5$. One obvious improvement of the model, is the implementation of the dipole size evolution based on the Green function. The consequence will be a smaller suppression, particularly at $z=1$. 

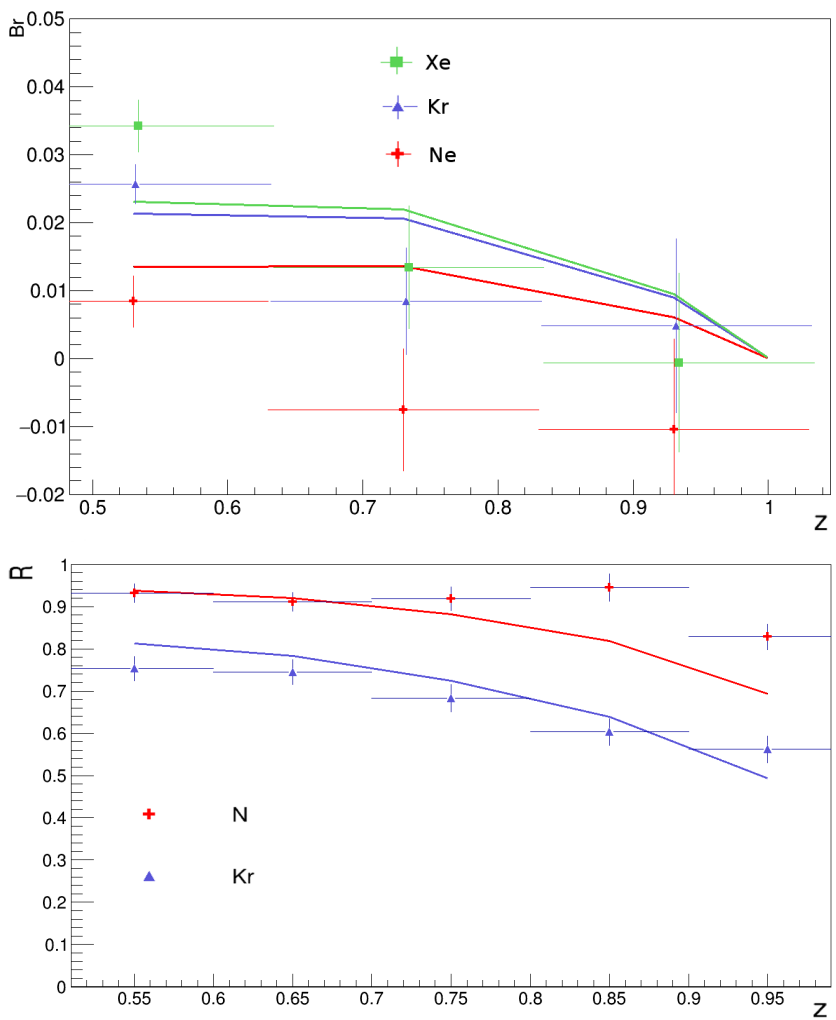

Figure 4. Top : $p_{t}$-broadening compared to HERMES data [3]. bottom : multiplicity ratio taking into account induced energy loss.

\section{Acknowledgment}

B. G. gratefully acknowledges generous support from Chilean FONDECYT grants 3160493. B. G. acknowledges support by the Basal project FB0821.

\section{References}

[1] E.L. Berger, Phys. Lett. B 89, 241 (1980)

[2] HERMES Collaboration, A. Airapetian, et al., Phys. Lett. B 57737 (2003)

[3] HERMES Collaboration, A. Airapetian, et al., Phys. Lett. B 684114 (2010)

[4] F. Arleo, Eur. Phys. J. C 30213 (2003)

[5] E. Wang, X. N. Wang, Phys. Rev. Lett. 89162301 (2002)

[6] EMC Collaboration, J. Ashman et al., Z. Phys. C 521 (1991);

EMC Collaboration, M. Arneodo et al., Nucl. Phys. B 321541 (1989)

[7] K. Gallmeister and U. Mosel, Nucl.Phys. A 80168 (2008)

[8] B. Z. Kopeliovich et al., Nuclear Physics A 740211 (2004)

[9] B. Z. Kopeliovich, H. J. Pirner, I.K. Potashnikova, I. Schmidt, Physics Letters B 662117 (2008) 
[10] Y. Dokshitzer, Phil.Trans.Roy.Soc.Lond.A 359309 (2001)

[11] H. DE VRIES, C. W. DE JAGER and C. DE VRIES, ATOMIC DATA AND NUCLEAR DATA TABLES 36 495-536 (1987)

[12] B.Z. Kopeliovich, A. Schäfer, and A.V. Tarasov, Phys. Rev. D 62054022 (2000)

[13] B.Z. Kopeliovich, J. Nemchik, A. Schäfer, A.V. Tarasov, Phys. Rev. C 65 (2002) 035201

[14] R.M. Barnett, et al., Phys. Rev. D 54, 191 (1996)

[15] I. Strakovsky, total and total elastic cross sections for $\pi^{+} p$ collision, private communication

[16] S.R. Amendolia, et al., Phys. Lett. B 178435 (1986)

[17] M.B. Johnson, B.Z. Kopeliovich and A.V. Tarasov, Phys. Rev. C 6335203 (2001)

[18] R. Baier, Yu.L. Dokshitzer, A.H. Mueller, S. Peigne and D. Schiff, Nucl. Phys. B484 265 (1997) 